\title{
Quetiapine $v$. lithium in the maintenance phase following a first episode of mania: randomised controlled trial
}

Michael Berk, Rothanthi Daglas, Orwa Dandash, Murat Yücel, Lisa Henry, Karen Hallam, Craig Macneil, Melissa Hasty, Christos Pantelis, Brendan P. Murphy, Linda Kader, Saji Damodaran, Michael T. H. Wong, Philippe Conus, Aswin Ratheesh, Patrick D. McGorry and Sue M. Cotton

\section{Background}

Lithium and quetiapine are considered standard maintenance agents for bipolar disorder yet it is unclear how their efficacy compares with each other

\section{Aims}

To investigate the differential effect of lithium and quetiapine on symptoms of depression, mania, general functioning global illness severity and quality of life in patients with recently stabilised first-episode mania.

\section{Method}

Maintenance trial of patients with first-episode mania stabilised on a combination of lithium and quetiapine, subsequently randomised to lithium or quetiapine monotherapy (up to $800 \mathrm{mg} /$ day) and followed up for 1 year (Trial registration: Australian and New Zealand Clinical Trials Registry - ACTRN12607000639426.)

\section{Results}

In total, 61 individuals were randomised. Within mixed-model repeated measures analyses, significant omnibus treatment $\times$ visit interactions were observed for measures of overall psychopathology, psychotic symptoms and functioning. Planned and post hoc comparisons further demonstrated the superiority of lithium treatment over quetiapine.

\section{Conclusions}

In people with first-episode mania treated with a combination of lithium and quetiapine, continuation treatment with lithium rather than quetiapine is superior in terms of mean levels of symptoms during a 1-year evolution.

\section{Declaration of interest}

This study was supported by an unrestricted grant from AstraZeneca. R.D., M.H. and K.H. worked on a study that has received research support by AstraZeneca. M.B. has received grant/research support from the $\mathrm{NIH}$, Cooperative Research Centre, Simons Autism Foundation, Cancer Council of Victoria, Stanley Medical Research Foundation, MBF, NHMRC, Beyond Blue, Rotary Health, Geelong Medical Research Foundation, Bristol Myers Squibb, Eli Lilly, Glaxo Smithkline, Meat and Livestock Board, Organon, Novartis, Mayne Pharma, Servier and Woolworths, has been a speaker for AstraZeneca, Bristol Myers Squibb, Eli Lilly, Glaxo SmithKline, Janssen-Cilag, Lundbeck, Merck, Pfizer, Sanofi Synthelabo, Servier, Solvay and Wyeth, and served as a consultant to AstraZeneca, Bioadvantex, Bristol Myers Squibb, Eli Lilly, Glaxo Smithkline, Janssen-Cilag, Lundbeck Merck and Servier. C.M. has received a grant/ research support from Eli Lilly and AstraZeneca, Janssen Cilag and Sanofi Aventis, and served as a consultant to AstraZeneca and Eli Lilly. P.D.M. has received investigator initiated research grants from AstraZeneca, Janssen-Cilag, Eli Lilly and BMS. He has received honoraria for educational events from Janssen-Cilag, Eli Lilly, BMS, AstraZeneca, Pfizer and Lundbeck. C.P. has participated on Advisory Boards for Janssen-Cilag, AstraZeneca, Lundbeck and Servier, has received honoraria for talks presented at educational meetings organised by AstraZeneca, Janssen-Cilag, Eli-Lilly, Pfizer, Lundbeck and Shire.

\section{Copyright and usage}

(c) The Royal college of Psychiatrists 2017.
Kraepelin noted that with cumulative episodes of illness, the interepisode gap of subsequent episodes shortened. ${ }^{1}$ Post later coined the term 'kindling' to describe a neurobiological model of sensitisation whereby recurrence primes neurobiology towards greater vulnerability, although computational artefact may play a role in this phenomenon. ${ }^{2,3}$ The consequent staging model has inherent and testable hypotheses, including a differential effect of treatments at different illness stages. ${ }^{4}$ There are hints that lithium may be most effective early in the disease course, ${ }^{5,6}$ with a suggestion of loss of efficacy following discontinuation and reinstitution, ${ }^{7}$ although not all reports support this notion. ${ }^{8}$ Atypical antipsychotic agents, ${ }^{9}$ have efficacy in acute mania, ${ }^{10-12}$ with quetiapine demonstrating efficacy in bipolar depression. ${ }^{13,14}$ Atypical antipsychotic agents also show mood stabilising effects in maintenance trials. ${ }^{15-17}$ There is tentative evidence that atypical antipsychotics also demonstrate greater efficacy earlier in the disease course. ${ }^{18}$ However, trials in early-stage illness are lacking. This study focused on the comparative efficacy on symptomatic recovery of two current first-line clinical choices, lithium and quetiapine, among a group of young people with first-episode mania with prevalent psychotic features. The aim was to assess whether lithium and quetiapine put head to head provided comparable levels of symptomatic recovery on measures of depression, mania, clinical global impression and overall symptomatic severity. These are the secondary outcome measures of our trial (registered with the Australian and New Zealand Clinical Trials Registry - ACTRN12607000639426). Further, the study aimed to explore whether these medications differed in their effectiveness in improving functional outcomes in the 12 months following a first manic episode with psychotic features. 


\section{Method}

\section{Study design}

This single-blind controlled randomised parallel group design was conducted over 52 weeks at two sites in Melbourne, Australia; Orygen, The National Centre of Excellence in Youth Mental Health, the Early in Life Mental Health Service (ELMHS) and the Recovery and Prevention of Psychosis (RAPP) services at Monash Health. All individuals presenting with an acute first episode of mania with psychotic features were stabilised on a combination of quetiapine plus lithium in an open manner as part of a routine care protocol. Following informed consent, they were randomised after remission (based on clinical assessment by treating clinicians, a period of between 2 to 3 months) to either lithium or quetiapine - this was the baseline for analyses. The study assessed recovery from first episode during the 12-month period on symptomatic, quality of life and functional outcome scales. Neuroanatomical changes and neuropsychological functioning were the primary outcomes - these data are presented elsewhere. The trial was conducted according to Good Clinical Practice (GCP) guidelines and approved by all relevant ethical committees for services involved.

\section{Participants}

Patients meeting DSM-IV-TR criteria for a manic episode on the Structured Clinical Interview for DSM-IV-TR - Patient Edition $(\mathrm{SCID}-\mathrm{I} / \mathrm{P})^{19}$ were recruited between December 2008 and December 2013. To facilitate generalisability and because of diagnostic instability at the early stages of illness, the trial had broad inclusion criteria. Individuals with first-episode mania aged 15 to 25 years were included. Individuals meeting SCID-I/P criteria for bipolar I disorder, substance-induced mood disorder or schizoaffective disorder were eligible. Other inclusion criteria included: a Young Mania Rating Scale (YMRS) ${ }^{20}$ score of at least 20 for the acute phase of a first episode of mania (the YMRS was administered as a part of clinical care on these units); no previously treated manic episode(s); having the capacity to provide informed consent for the study and comply with study procedures; be utilising effective contraception if female. Even though psychosis was not an inclusion criterion, because triage selects for severity, it was present in most participants. Patients had to have been on quetiapine and lithium as standard therapy for at least 1 month prior to randomisation.

Exclusion criteria from the trial included: patients with a known or suspected clinically relevant systemic medical disorder; pregnant or lactating females; patients who had a prior sensitivity or allergy to quetiapine, lithium or their components; inability to comply with either the requirements of informed consent or the treatment protocol; non-fluency in English; history of epilepsy; clinically relevant biochemical or haematological abnormalities at baseline; patients at immediate risk of self-harm or risk to others; organic mental disease, including intellectual disability (full scale IQ $<70$ ); and an absolute neutrophil count of $\geqslant 1.5 \times 10^{9}$ per litre. Use of cytochrome P450 3A4 inhibitors or P450 inducers was not permitted in the 14 days preceding enrolment. Extra exclusion criteria applied to individuals with diabetes mellitus: unstable diabetes mellitus defined as enrolment glycosylated haemoglobin (HbAlc) $>8.5 \%$; admission to hospital for treatment of diabetes or diabetes-related illness in the previous 12 weeks; not under physician care for diabetes; physician responsible for patient's diabetes care did not indicate that patient's diabetes was controlled; physician responsible for patient's diabetes care did not approve patient's participation in the study; had not been on the same dose of oral hypoglycaemic $\operatorname{drug}(\mathrm{s})$ for the 4 weeks prior to randomisation ( 8 weeks for thiazolidinediones); daily insulin had been more than $10 \%$ outside their mean monthly dose on one or more occasions in the preceding 4 weeks.

All individuals presenting with an acute first manic episode with psychotic features were acutely stabilised on a combination of quetiapine plus lithium in an open manner as part of a routine care protocol. Individuals received quetiapine at a dose determined by the treating clinician. Optimal serum lithium levels between 0.8 and $1.0 \mathrm{mmol} / \mathrm{L}$ were targeted. Following clinical stabilisation (based on the global impression of the treating clinician or team) and provision of written informed consent, participants were randomised to either quetiapine or lithium. Discontinuation occurred very gradually over weeks or months at the discretion of treating clinicians. Lithium levels of $0.6-0.8 \mathrm{mmol} / \mathrm{L}$ were targeted in the maintenance phase and the quetiapine dose was determined by the treating clinician.

\section{Randomisation}

An independent statistician generated a computerised randomisation sequence. Randomisation and consent took place around months 2-3 following stabilisation from a first episode of mania, at the discretion of the treating team. A randomisation log was established, and a set of sequentially ordered envelopes was kept in a locked filing cabinet at the Orygen Research Centre site.

\section{Masking}

The patients, treating psychiatrist and case managers knew which treatment the patient was receiving whereas research assistants, neuropsychologists and all individuals involved in neuroimaging, analysis and data management remained masked to this information. In particular, participants were told not to communicate information regarding treatment to the research assistants and imaging personnel by the study clinicians, and no information regarding treatment was communicated at team meetings or was included in the study files to which the research assistants had access.

\section{Measurements}

Participants were assessed at baseline using the SCID-I/P. ${ }^{19}$ Clinical assessments were carried out at baseline and at fortnightly intervals for the first month, then on a monthly basis for the following 2 months and then at 3-monthly intervals thereafter concluding at the 12-month time point. The patient's psychiatric condition was further assessed using the Brief Psychiatric Rating Scale (BPRS), ${ }^{21}$ Bipolar Depression Rating Scale (BDRS), ${ }^{22}$ Montgomery-Åsberg Depression Rating Scale (MADRS), ${ }^{23}$ YMRS, $^{20}$ Clinical Global Impressions modified for bipolar disorder (CGI-BP) ${ }^{24}$ Global Assessment of Functioning (GAF) scale, ${ }^{25}$ Social and Occupational Functioning Assessment Scale $(\text { SOFAS) })^{26}$ and Quality of Life Scale ${ }^{27}$ (online Table DS1). Laboratory values were undertaken at baseline and when clinically indicated. Lithium monitoring was done according to Orygen and Monash Health protocols. In order to screen for neutropenia, monthly blood counts (complete blood counts) with a white blood cell differential count for the first 3 months and then quarterly thereafter, were completed throughout the study. Additionally, to screen for diabetes mellitus, fasting plasma glucose and $\mathrm{HbAlc}$ were measured at randomisation and every 12 weeks.

If a participant experienced a psychotic, depressive or manic relapse requiring admission during the course of the follow-up 
period (12 months), the research interviewer conducted a relapse assessment with the participant and/or clinician. The relapse assessment included the time to intervention for a mood episode (TIME, defined as the time to any clinical intervention in response to a mood episode), YMRS, BDRS, BPRS, MADRS, CGI-BP, GAF/ SOFAS, Vocation \& Location Index. ${ }^{28}$

\section{Recruitment procedure and withdrawal criteria}

When potential participants presented with symptoms compatible with a manic episode with psychotic features, the mania study team was contacted for an assessment. Participants were managed for $24-48 \mathrm{~h}$ with holding treatment, usually benzodiazepines, in order to confirm the diagnosis and exclude a transient state induced by intoxication. All individuals were treated acutely with combination quetiapine and lithium, as algorithm-based standard clinical practice. To aid feasibility and generalisability, there were no exclusions in terms of concomitant medications. Withdrawal from the trial occurred if participants became non-adherent with medication, withdrew their consent to participate, became pregnant or developed serious adverse events to the medication.

\section{Data analysis}

All analyses were conducted in accordance with the International Conference on Harmonisation E9 statistical principles (ICH-E9). Independent samples $t$-tests and chi-squared $\left(\chi^{2}\right)$ analyses were used to test for differences between the two treatment groups at baseline. These inferential statistics were also used to compare participants who completed/discontinued the treatments. All randomised participants who had at least one post-baseline assessment were included in the intent-to-treat analysis. Differences between the two treatment groups with respect to manic symptoms, depressive symptoms, psychotic symptoms, global psychopathology, functioning and score on the QLS were assessed using the likelihood based mixed-effects model repeated measures approach (MMRM). The MMRM model included the fixed, categorical effects of treatment group, visit and treatment group $\times$ visit interaction. The MMRM includes all available data at each time point ${ }^{29}$ and is the favoured approach for analysis of data from clinical trials in psychiatry. ${ }^{30}$ The Toeplitiz covariance structure was used to model the relations between observations on different occasions. Planned comparisons ( $t$-tests) using MMRM were conducted to examine treatment group differences in mean change on the outcome measures from baseline (week 0) to the primary endpoint of 12 months. Post hoc analyses were also conducted to determine whether change within treatment groups between baseline and 12 months were significant, and to delineate at which time points the two treatment groups differed significantly. No adjustments were made for multiple comparisons because they can result in a higher type II rate, reduced power and increased likelihood of missing important findings. ${ }^{31}$

\section{Results}

A total of 286 individuals were screened, of which 207 were deemed ineligible, most commonly because they had not been started on study medications $(n=106)$. Other reasons included a YMRS score less than $20(n=40)$, non-adherence with treatment $(n=15)$ and early discharge from the service $(n=27)$ (see Fig. 1 for further details). A total of 18 individuals declined to participate.

A total of 61 individuals were randomised. Further exclusions immediately followed (Fig. 1) for reasons that included (a) patient allocated to a non-preferred medication, as some participants had a preference for one or the other $(n=4)$; (b) disengagement from treatment $(n=4)$; (c) all medications ceased $(n=3)$; (d) clinically unstable and not suitable for randomisation $(n=2)$; and (e) relapsed prior to baseline $(n=2)$ or monotherapy $(n=3)$; and (f) withdrawn by clinician because of medication side-effects $(n=2)$. As a result there were 20 patients in the quetiapine group and 21 in the lithium group.

Baseline demographic characteristics of the 41 patients are detailed in online Table DS1. The ages ranged from 17 to 26 (mean 21.3, s.d. $=2.3$ ) with $32(78 \%)$ male. Most were single and living with their family of origin. The average level of education was 11.8 years $($ s.d. $=1.6)$. Just over $40 \%(n=18)$ of the cohort were unemployed and $29.3 \%(n=12)$ were students. The primary source of financial support was government benefits. There were no differences between the quetiapine and lithium groups on any of these demographic measures. On entry to the trial, participants were largely euthymic $(82.9 \%, n=34)$. There were no significant baseline differences on clinical, functioning and QLS measures for the total cohort as well as separately for the two treatment groups.

At 12 months, there were 11 participants who were lost to follow-up (Fig. 1, $n=5$ in the quetiapine and $n=6$ in the lithium groups). Reasons included withdrawal of consent $(n=3)$ and unavailable/non-contactable $(n=3)$. Two participants had a relapse or were admitted as in-patients, one patient reported an adverse event, one patient moved overseas and another failed to comply with monotherapy protocol. One person in the quetiapine group had no post-baseline data and was excluded from intention-to-treat (ITT) analysis. The remainder of the 10 participants had some post-baseline data and were included in the MMRM. Thus, data were available for 40 participants (quetiapine, $n=19$; lithium $n=21$ ) (Fig. 1).

There was no significant difference between the medication groups with respect to completion of assessments at 12 months, $\chi^{2}(1)=0.07, P=0.796$. The individuals who were non-completers were significantly more likely to be depressed at baseline (MADRS non-completers mean 12.91 (s.d.=10.66); completers mean 5.37 (s.d.=7.44), $\quad t(39)=2.37, \quad P=0.023$ : CGI-BP, depression noncompleters mean 3.00 (s.d.=1.94); completers mean 1.70 (s.d.=1.26), $t(39)=2.44, P=0.019)$. The CGI-BP severity score was also significantly greater in the non-completers (mean 3.00, s.d. $=1.84$ ) as compared with completers (mean 1.67 , s.d. $=1.18$, $t(13.85)=2.20, P=0.045$; degrees of freedom was adjusted to take into account heterogeneity in variances in the two groups). Figure 2 demonstrates the mean estimates based on untransformed data of the symptom and functioning measures over the 12-month period.

\section{Manic symptoms}

For the YMRS, there was no significant interaction between treatment group and visit, and the degree of change from baseline to 12 months did not differ significantly between the two treatment groups (Tables 1 and 2). For the CGI-BP mania subscale, there was a significant interaction between treatment group and visit, $F(7,112.9)=2.48, P=0.021$; but the degree of change between baseline to 12 months did not differ between the two groups, $P=0.069$. Post hoc analyses indicated that the quetiapine group (mean 2.0, s.d. $=0.2$ ) had a significantly higher mean CGI-BP mania score than the lithium group (mean 1.1, s.d. $=0.2)$ at 9 months $(P=0.001)$.

\section{Depressive symptoms}

On measures of depression, the omnibus interactions between treatment group and visit were not significant for both the BDRS and MADRS (Table 1). However, the two treatment groups differed with respect to the degree of change on the MADRS 

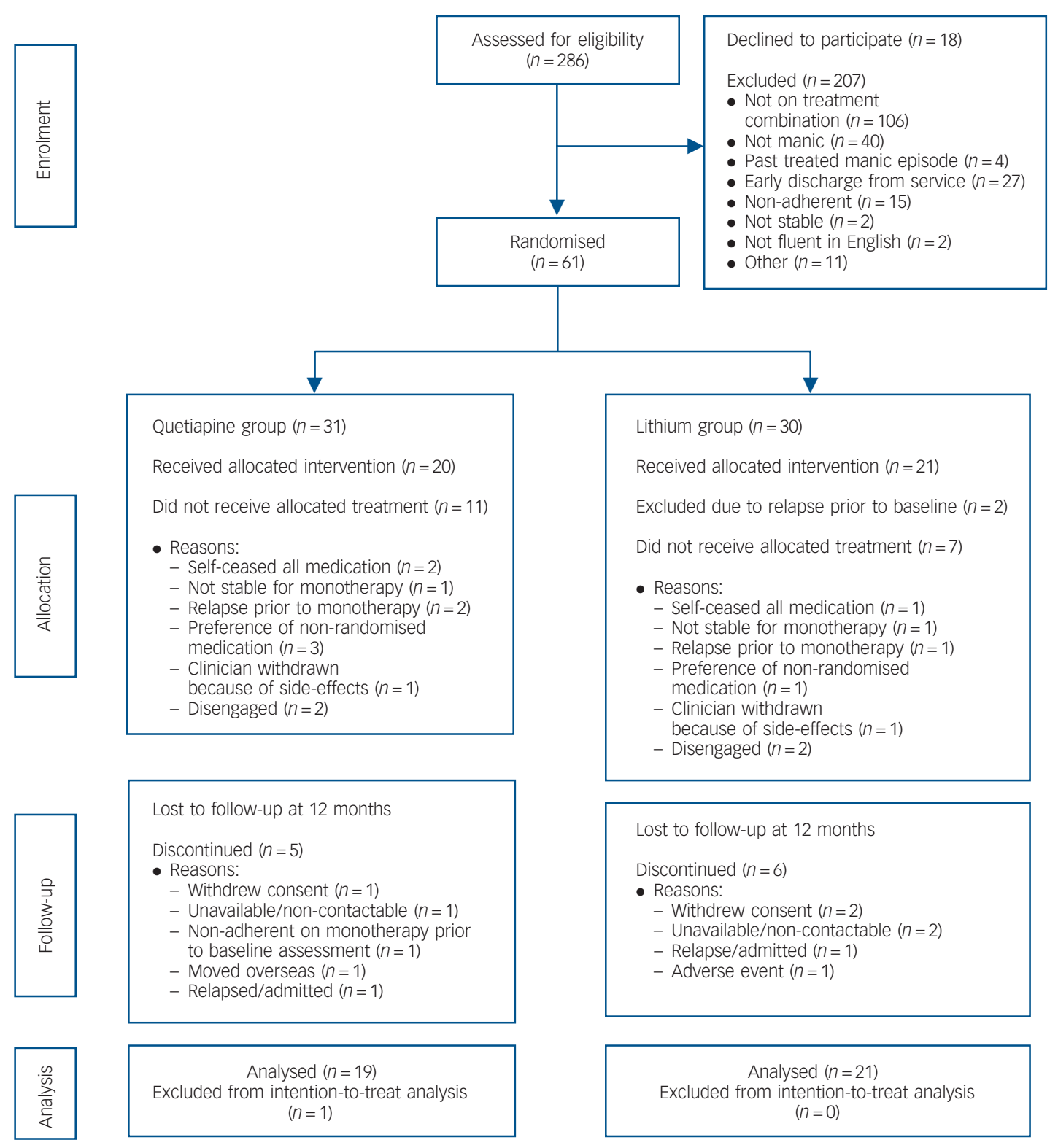

Fig. 1 CONSORT flow chart.

between baseline and 12 months, $t(39.1)=-2.88, P=0.006$ (Table 2). Post hoc analyses revealed that the quetiapine group had a significant worsening of depressive symptoms from baseline to week 52 (baseline mean 7.1, s.d.=1.7; week 52 mean 13.4, s.d. $=1.8, P=0.028)$, whereas no such change was observed for the lithium group (baseline mean 6.9, s.d.=1.6; week 52 mean 2.5 , s.d. $=1.8, P=0.085$ ). Furthermore, at 52 weeks, post hoc comparisons indicated that the quetiapine group had significantly higher levels of depression as measured by the MADRS as compared with the lithium group ( $P=0.001$; Table 2 and Fig. 2$)$.

\section{Positive psychotic symptoms}

The omnibus interaction between group and visit was significant for the BPRS psychosis subscales, $F(7,104.2)=2.13, P=0.047$, and the degree of change from baseline to 12 months was significantly greater in the quetiapine $v$. lithium groups, $t(57.9)=-2.99$, $P=0.004$ (Tables 1 and 2). Post hoc analyses indicated that positive symptoms worsened from baseline (mean 4.3, s.d.=0.4) to 12 months (mean 6.1, s.d. $=0.5$ ) in the quetiapine group
$(P=0.003)$, whereas there were no such change in the lithium group (baseline mean 4.6, s.d. $=0.4$; week 52 mean 4.3, s.d. $=0.5, P=0.292)$. At 12 months, the quetiapine group had significantly greater levels of positive symptoms than the lithium group ( $P=0.005$; Table 2 and Fig. 2$)$.

\section{Overall psychopathology}

On the BPRS total score, there was a significant interaction between treatment group and visit, $F(7,72.7)=3.25, P=0.005$ (Table 1). Planned comparisons indicated that the degree of change in BPRS total score from baseline to 12 months differed significantly between the two groups, $t(50.2)=-3.61, P=0.001$ (Table 2). There was a statistically significant worsening in BPRS scores from baseline (mean 32.5, s.d. $=2.0$ ) to 12 months (mean 39.6 , s.d. $=2.3)$ in the quetiapine group $(P=0.008)$, whereas a significant decline in symptoms was observed in the lithium group (baseline mean 33.4, s.d. $=1.9 ; 52$ weeks mean 28.1, s.d. $=2.2$, $P=0.023$ ). At both 36 weeks (quetiapine mean 34.8 , s.d. $=2.5$; lithium mean 27.3, s.d. $=2.0, P=0.040$ ) and 52 weeks (quetiapine 
Table 1 Tests of fixed effects in mixed-effects repeated measures models (MMRM) for measures of psychopathology, functioning and quality of life

\begin{tabular}{|c|c|c|}
\hline Effect & F-test (d.f.) & $P$ \\
\hline \multicolumn{3}{|l|}{ Mania } \\
\hline \multicolumn{3}{|c|}{ Young Mania Rating Scale ${ }^{a}$} \\
\hline Treatment & $0.12(1,38.8)$ & 0.728 \\
\hline Visit & $1.26(7,86.3)$ & 0.277 \\
\hline Treatment $\times$ visit & $1.39(7,88.3)$ & 0.218 \\
\hline \multicolumn{3}{|c|}{ Clinical Global Impressions - Bipolar, mania ${ }^{a}$} \\
\hline Treatment & $1.13(1,35.8)$ & 0.295 \\
\hline Visit & $2.28(7,112.9)$ & 0.033 \\
\hline Treatment $\times$ visit & $2.48(7,112.9)$ & 0.021 \\
\hline \multicolumn{3}{|l|}{ Depressive symptoms } \\
\hline \multicolumn{3}{|c|}{ Bipolar Depression Rating Scale ${ }^{a}$} \\
\hline Treatment & $3.91(1,37.2)$ & 0.055 \\
\hline Visit & $3.44(7,88.2)$ & 0.003 \\
\hline Treatment $\times$ visit & $1.49(7,88.2)$ & 0.180 \\
\hline \multicolumn{3}{|c|}{ Montgomery-Åsberg Depression Rating Scale ${ }^{a}$} \\
\hline Treatment & $3.44(1,35.8)$ & 0.072 \\
\hline Visit & $1.94(7,68.6)$ & 0.076 \\
\hline Treatment $\times$ visit & $2.06(7,68.6)$ & 0.060 \\
\hline \multicolumn{3}{|c|}{ Clinical Global Impressions - Bipolar, depression ${ }^{a}$} \\
\hline Treatment & $5.56(1,32.8)$ & 0.024 \\
\hline Visit & $1.74(7,56.9)$ & 0.119 \\
\hline Treatment $\times$ visit & $2.05(7,56.9)$ & 0.064 \\
\hline \multicolumn{3}{|l|}{ Psychotic symptoms } \\
\hline \multicolumn{3}{|c|}{ Brief Psychiatric Rating Scale, positive symptoms ${ }^{a}$} \\
\hline Treatment & $0.71(1,27.1)$ & 0.408 \\
\hline Visit & $1.52(7,104.2)$ & 0.170 \\
\hline Treatment $\times$ visit & $2.13(7,104.2)$ & 0.047 \\
\hline \multicolumn{3}{|c|}{ Overall psychopathology } \\
\hline \multicolumn{3}{|c|}{ Brief Psychiatric Rating Scale ${ }^{a}$} \\
\hline Treatment & $1.84(1,34.7)$ & 0.184 \\
\hline Visit & $2.31(7,72.7)$ & 0.035 \\
\hline Treatment $\times$ visit & $3.25(7,72.7)$ & 0.005 \\
\hline \multicolumn{3}{|c|}{ Clinical Global Impressions - Bipolar, severity ${ }^{a}$} \\
\hline Treatment & $7.04(1,32.6)$ & 0.012 \\
\hline Visit & $2.99(7,87.2)$ & 0.007 \\
\hline Treatment $\times$ visit & $3.10(7,87.2)$ & 0.006 \\
\hline \multicolumn{3}{|l|}{ Functioning } \\
\hline \multicolumn{3}{|c|}{ Global Assessment of Functioning } \\
\hline Treatment & $1.10(1,37.9)$ & 0.302 \\
\hline Visit & $1.56(7,70.9)$ & 0.161 \\
\hline Treatment $\times$ visit & $4.12(7,70.9)$ & 0.001 \\
\hline \multicolumn{3}{|c|}{$\begin{array}{l}\text { Social and Occupational Functioning Assessment } \\
\text { Scale }\end{array}$} \\
\hline Treatment & $1.75(1,39.7)$ & 0.193 \\
\hline Visit & $1.84(7,65.9)$ & 0.095 \\
\hline Treatment $\times$ visit & $3.92(7,65.9)$ & 0.001 \\
\hline \multicolumn{3}{|l|}{ Quality of life } \\
\hline \multicolumn{3}{|l|}{ Quality of Life Scale } \\
\hline Treatment & $1.37(1,38.1)$ & 0.248 \\
\hline Visit & $0.70(2,41.5)$ & 0.505 \\
\hline Treatment $\times$ visit & $2.81(2,41.5)$ & 0.072 \\
\hline
\end{tabular}

a. Because of skewness, logarithmic transformation (plus constant) was performed. MMMR was based on the transformed data.

mean 39.6, s.d. =2.3; lithium mean 28.1, s.d. $=2.2, P<0.001$ ), the quetiapine group had significantly higher scores on CGI-BP severity than the lithium group (Table 2 and Fig. 2).

On the CGI-BP, severity, the omnibus interaction was significant, $F(7,87.2)=3.10, P=0.006$ (Table 1). Again the degree of change from baseline to 12 months differed significantly between the two groups, $t(73.9)=-3.98, P<0.001$ (Table 2 ). Post hoc analysis indicated that the quetiapine group had a worsening of symptoms from baseline to 12 months (baseline mean 1.9, s.d. $=0.3 ; 52$ weeks mean 3.6, s.d. $=0.3, P<0.001$ ), whereas no such change was observed for the lithium group (baseline mean
2.0 , s.d. $=0.3 ; 52$ weeks mean 1.3 , s.d. $=0.3, P=0.052$ ). At both 36 weeks (quetiapine mean 2.4, s.d. $=0.4$; lithium mean 1.2, s.d. $=0.3, \quad P=0.010$ ) and 52 weeks (quetiapine mean 3.6, s.d. $=0.3$; lithium mean 1.3 , s.d. $=0.3, P<0.001)$, the quetiapine group had significantly higher scores on CGI-BP severity than the lithium group (Table 2 and Fig. 2).

\section{Functioning and quality of life}

For both the GAF $(F(7,70.9)=4.12, P=0.001)$ and SOFAS $(F(7,65.9)=3.92, P<0.001)$, there were significant interactions between treatment group and visit (Table 1). On both of these measures, the degree of change from baseline to 12 months differed significantly between the two groups (GAF, $t(38.3)=3.39, P=0.002$; SOFAS, $t(40.1)=2.88, P=0.006$ ) (Table 2). Post hoc analyses indicated that the quetiapine group had a significant drop in functioning from baseline to 12 months (GAF, baseline mean 68.7 , s.d. $=3.5 ; 52$ weeks mean 57.0 , s.d. $=3.8, P=0.008$; SOFAS, baseline mean 67.7, s.d. $=3.6 ; 52$ weeks mean 57.2, s.d. $=3.9$, $P=0.020$ ), whereas functioning remained stable in the lithium group (GAF, baseline mean 69.1, s.d. $=3,3 ; 52$ weeks mean 77.0, s.d. $=3.7, P=0.057$; SOFAS, baseline mean 70.8, s.d. $=3.4 ; 52$ weeks mean 77.6 , s.d. $=3.8, P=0.109)$. At 12 months, functioning was significantly lower in the quetiapine compared with the lithium group (GAF $P<0.001$; SOFAS $P<0.001$ ). Significant differences in global functioning between the two groups on the GAF were also seen at 9 months (quetiapine mean 67.2, s.d. $=4.0$; lithium, mean 78.5, s.d. $=3.5, P=0.038$; Fig. 2). For the QLS, there was no significant interaction between treatment group and visit, $F(2,41.5)=2.81, P=0.072$. There was also no between-group difference in degree of change from baseline to 12 months.

\section{Medications}

The mean lithium level in the lithium group was $0.6 \mathrm{mmol} / \mathrm{L}$, and the mean quetiapine dose was $437.5 \mathrm{mg}$. Three participants in both the quetiapine and lithium groups were given antidepressants during the maintenance phase of the trial (Fisher's exact test, $P=1.000$ ). Five participants in the quetiapine and two in the lithium group received benzodiazepines during the maintenance phase (Fisher's exact test, $P=0.225$ ).

\section{Discussion}

\section{Main findings and comparison with findings from other studies}

This study in a group of patients naturalistically treated with lithium and quetiapine for a first manic episode with psychotic features, assessed whether discontinuation of one or the other provided comparable levels of symptomatic recovery over 12 months on measures of depression, mania, psychosis and overall illness severity, and whether these medications were associated with differences in functioning and quality of life. In terms of depression, mania and psychotic symptoms on the BDRS, MADRS and YMRS and functional outcomes as rated by clinicians, lithium provided better recovery. These differences between baseline and 12 months were also evident on measures of overall psychopathology such as the BPRS and CGI-BP, as well as functioning on measures such as the GAF and SOFAS, but not on patient-rated quality of life.

These are surprising findings for a number of reasons, not least the failure of comparable studies to find substantive differences between atypical antipsychotics and conventional mood stabilisers in maintenance. ${ }^{32}$ They contrast with the 


\begin{tabular}{|c|c|c|c|c|}
\hline \multirow[b]{2}{*}{ Characteristics } & \multicolumn{4}{|c|}{ Change from baseline to week 52} \\
\hline & $\begin{array}{l}\text { Quetiapine, mean } \\
(\text { s.e. })^{\mathrm{a}}\end{array}$ & $\begin{array}{l}\text { Lithium, mean } \\
\text { (s.e.) }\end{array}$ & $t^{\mathrm{b}}$ (d.f.) & $P$ \\
\hline \multicolumn{5}{|l|}{ Mania } \\
\hline $\begin{array}{l}\text { Young Mania Rating Scale } \\
\text { Clinical Global Impressions - Bipolar, mania }{ }^{c}\end{array}$ & $\begin{array}{l}-4.6(1.8) \\
-0.7(0.2)\end{array}$ & $\begin{array}{r}0.5(1.8) \\
-0.0(0.2)\end{array}$ & $\begin{array}{l}-1.61(65.4) \\
-1.84(107.8)\end{array}$ & $\begin{array}{l}0.113 \\
0.069\end{array}$ \\
\hline \multicolumn{5}{|l|}{ Depression } \\
\hline Bipolar Depression Rating Scale ${ }^{c}$ & $-4.0(2.2)$ & $3.7(2.1)$ & $-1.82(50.5)$ & 0.074 \\
\hline Montgomery-Åsberg Depression Rating Scale ${ }^{c}$ & $-6.4(2.2)$ & $4.4(2.1)$ & $-2.88(39.1)$ & 0.006 \\
\hline Clinical Global Impressions - Bipolar, depression ${ }^{c}$ & $-1.0(0.4)$ & $0.9(0.4)$ & $-3.20(54.2)$ & 0.002 \\
\hline \multicolumn{5}{|l|}{ Positive psychotic symptoms } \\
\hline Brief Psychiatric Rating Scale ${ }^{c}$ & $-1.7(0.7)$ & $0.5(0.6)$ & $-2.99(57.9)$ & 0.004 \\
\hline \multicolumn{5}{|l|}{ Overall psychopathology } \\
\hline Brief Psychiatric Rating Scale ${ }^{c}$ & $-7.1(2.6)$ & $5.3(2.5)$ & $-3.61(50.2)$ & 0.001 \\
\hline Clinical Global Impressions - Bipolar, severity ${ }^{c}$ & $-1.7(0.4)$ & $0.7(0.4)$ & $-3.98(73.9)$ & $<0.001$ \\
\hline \multicolumn{5}{|l|}{ Functioning } \\
\hline Global Assessment of Functioning & $11.7(4.2)$ & $-7.9(4.0)$ & $3.39(38.3)$ & 0.002 \\
\hline Social and Occupational Assessment Scale & $10.4(4.3)$ & $-6.8(4.1)$ & $2.88(40.1)$ & 0.006 \\
\hline \multicolumn{5}{|l|}{ Quality of life } \\
\hline Quality of Life Scale & $0.3(0.2)$ & $-0.3(0.2)$ & $1.58(29.6)$ & 0.124 \\
\hline
\end{tabular}

naturalistic CHOICE study, ${ }^{33}$ where quetiapine or lithium was added to other required treatments in individuals with largely chronic illness across the bipolar spectrum taking multiple medications experiencing mainly depressive symptoms. No marked differences between the treatment groups were seen. In contrast, the majority of patients in our study presented with psychotic features as part of a first-episode mania and a bipolar I diagnosis. In addition, the CHOICE study had a follow-up period of 6 months whereas statistically significant separation between agents was largely seen at the 9 and 12 month time points. It is possible that this time frame reflects a protective effect of lithium against the development of recurrence as opposed to maintenance effects after the index episode. ${ }^{33}$

Similarly, in an enriched maintenance design, 1226 participants following a manic, depressive or mixed episode were stabilised on open-label quetiapine and then randomised to continue quetiapine or to switch to either placebo or lithium. Both agents comparably prevented mood events. ${ }^{34}$ Suppes and colleagues compared quetiapine combined with lithium or valproate in bipolar I disorder maintenance in a pooled analysis. ${ }^{35}$ Participants $(n=1326)$ were given quetiapine plus lithium or divalproex before being randomised to either quetiapine or placebo plus lithium or divalproex. Quetiapine significantly lengthened the time to recurrence in a similar manner when added to either lithium or divalproex.

Explanations for these findings are open to conjecture. It is probable that patient selection criteria played a key role. First, a cohort who are largely in-patients with acute, severe and frequently psychotic illness may behave very differently to an out-patient cohort with more chronic and mild disorder - lithium may have advantages in the former group. Second, stage of illness may play a role, concordant with the conjecture that individuals with a first episode may have a differential response to treatment. ${ }^{5}$ Lithium was superior to carbamazepine among people who had not previously been exposed to mood stabilisers. ${ }^{36}$ Many major disorders seemingly display a neuroprogressive course, such that the neurocircuitry tasked with mood regulation is damaged by biochemical processes including decreased neurogenesis, inflammation, mitochondrial dysfunction, oxidative stress and apoptosis. $^{37,38}$ It is also possible that index polarity or predominant polarity play a role in determining medication response, with depressive and manic index polarity potentially differing in this regard. ${ }^{39,40}$ It is possible that patients in the lithium group are likely to adhere to treatment more because of blood monitoring compared with patients on quetiapine, although there were no data to confirm or refute this. Although both agents were discontinued in a similar manner, these findings may reflect differences in lithium and quetiapine withdrawal reactions. Finally, there is potentially influence of 'service filters'. The quality and ubiquity of primary care means that much that is easily treatable has already been treated, so academics in tertiary centres tend to see individuals whose condition is chronic and treatment refractory, and risk deducing that these disabling disorders are largely treatment non-responsive. This conjecture is reflected by data showing that drug-placebo differences have shrunk markedly across the past few decades across diverse disorders. ${ }^{41}$ A first episode is a largely unfiltered cohort, with the opportunity to see drug effects unmodified by service-filter effects.

\section{Limitations}

In interpreting these results, a number of methodological factors need also to be borne in mind. The low sample size was a consequence of the low incidence of the disorder, the requirement for acute treatment and stabilisation on a combination of lithium and quetiapine, and the difficulty for unwell clients to participate in a trial, which also contributed to attrition. The study was conducted in specialised youth mental health out-patient clinics and may not be generalisable to other treatment settings or patient populations in other countries. Use of concomitant medications and the inclusion of patients with substance-induced mania increases generalisability at the expense of risk of confounding. Results may not be generalisable to other atypical antipsychotic agents. The study did not use fixed doses of quetiapine, and although the lithium levels were in the recommended range, they were at the lower end. The study also included $12.2 \%(n=5)$ of individuals with schizoaffective disorder and substance-induced 
(a)

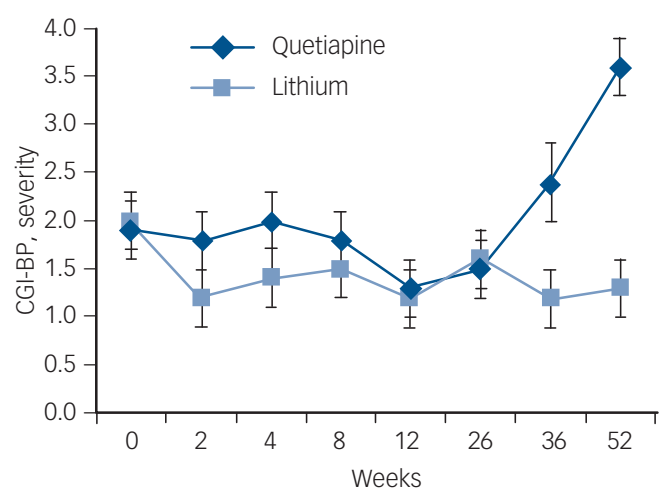

(c)

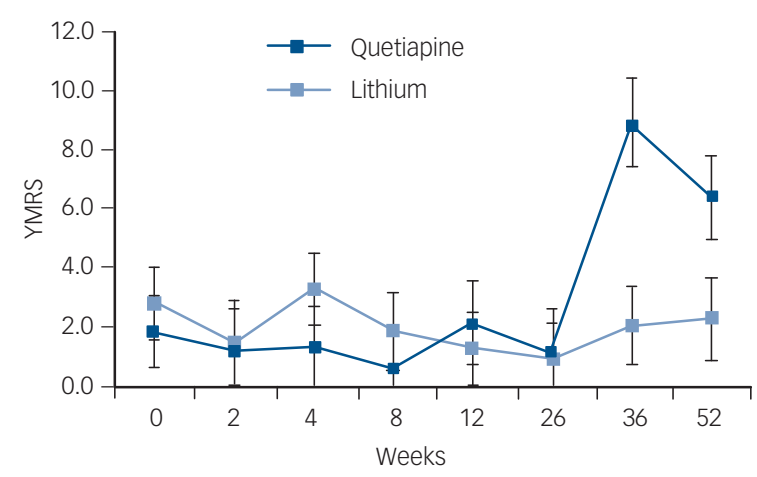

(e)

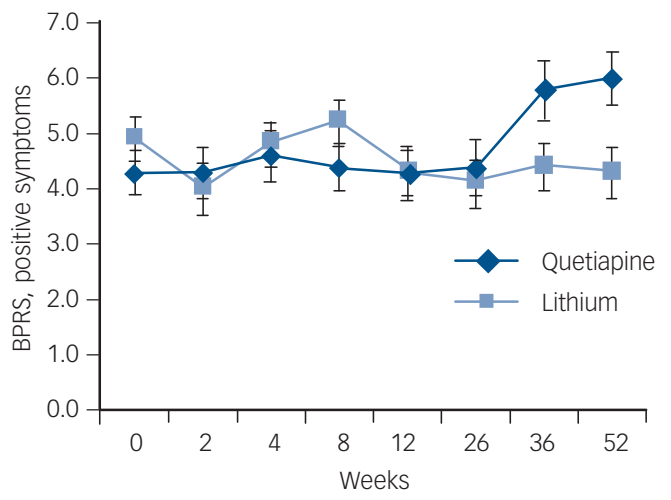

(g)

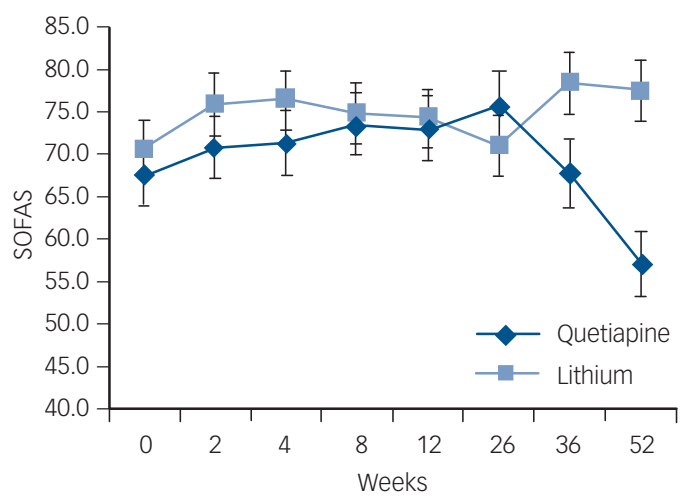

(b)

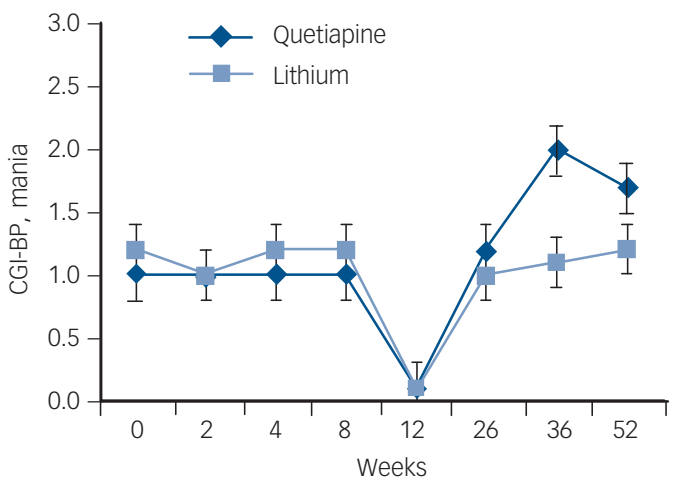

(d)

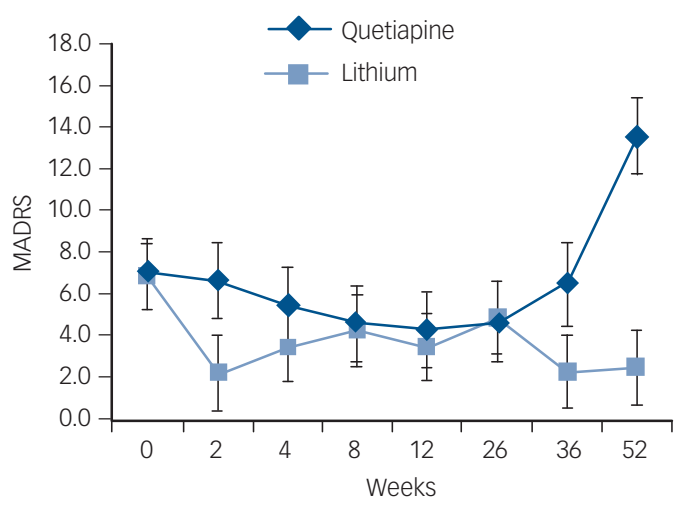

(f)

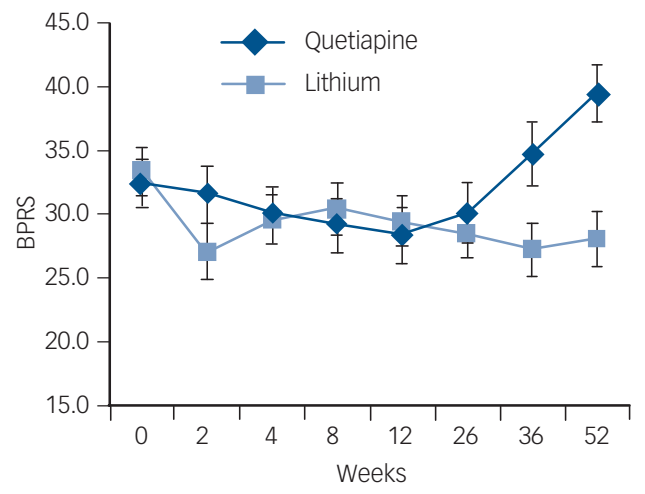

(h)

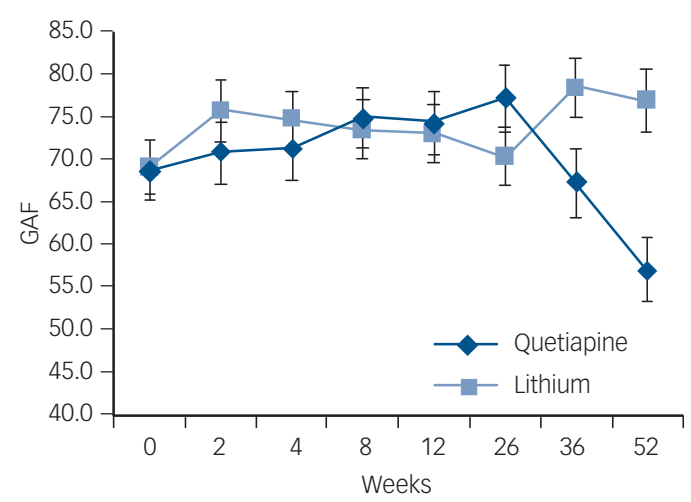

Fig. 2 Differences between quetiapine and lithium groups over a 12-month period (weeks baseline, 2, 4, 8, 12, 24, 36 and 52) on measures of mania, depression, psychopathology and psychotic symptoms as well as global functioning.

(a) Clinical Global Impressions - Bipolar (CGI-BP), severity; (b) CGI-BP, mania; (c) Young Mania Rating Scale (YMRS); (d) Montgomery-Åsberg Depression Rating Scale (MADRS); (e) Brief Psychiatric Rating Scale (BPRS), positive symptoms; (f) BPRS; (g) Social and Occupational Assessment Scale (SOFAS); (h) Global Assessment of Functioning (GAF). Error bars indicate standard deviation. 
mania, which may have influenced the results of the study, potentially favouring quetiapine. It is unknown in this population whether a combination of mood stabilisers is superior to a monotherapy for relapse prevention: a comparative study comparing monotherapy with combined treatment would be very informative. Strengths of the study include the relative homogeneity of the sample and the selection of a first-episode cohort. The study is also unique, being the first maintenance study in a first-episode cohort.

\section{Implications}

In conclusion, this study suggests that lithium was superior to quetiapine as maintenance therapy in terms of mean levels of symptoms in the year following a first episode of mania (after stabilisation on a combination of lithium plus quetiapine). This was an unexpected finding given the prior literature as well as the expected study power. The results reinforce the nascent literature that lithium might have greater efficacy in the early stages of the disorder, in those with more severe disorder and is similarly concordant with the differential efficacy of lithium in those with a manic index polarity. It reinforces lithium's role as a 'gold standard' of bipolar maintenance treatment concordant with recent reviews. ${ }^{42}$

Michael Berk, MBBCh, PhD, Deakin University, IMPACT Strategic Research Centre, School of Medicine, Barwon Health and the Geelong Clinic; Orygen, The National Centre of Excellence in Youth Mental Health, the Florey Institute for Neuroscience and Mental Health and the Department of Psychiatry, University of Melbourne, Victoria, Australia; Rothanthi Daglas, DPsych, Orygen, The National Centre of Victoria, Australia; Rothanthi Daglas, DPsych, Orygen, The National Centre of
Excellence in Youth Mental Health and the Centre for Youth Mental Health, University of Melbourne, Victoria, Australia; Orwa Dandash, PhD, Murat Yücel, PhD, Brain and Mental Health Laboratory, Monash Institute of Cognitive and Clinical Neurosciences (MICCN), School of Psychological Sciences, Monash University, Melbourne Neuropsychiatry Centre, Department of Psychiatry, University of Melbourne and Melbourne Health, Victoria, Australia; Lisa Henry, MPsych, Orygen, The National Centre of Excellence in Youth Mental Health and the Centre for Youth Mental Health, University of Melbourne, Victoria, Australia; Karen Hallam, PhD, Division of Psychology, School of Health and Biomedical Science, RMIT University, Melbourne, Victoria, Australia; Craig Macneil, DPsych, Melissa Hasty, DPsych, Orygen, The National Centre of Excellence in Youth Mental Health and the Centre for Youth Mental Health, University of Melbourne, Victoria, Australia; Christos Pantelis MD, Hon MD (Athens), Melbourne Neuropsychiatry Centre, Department of Psychiatry, University of Melbourne and Melbourne Health, Carlton South, Victoria, Australia: Brendan P. Murphy, FRCPsych, Department of Psychiatry, Faculty of Medicine, Nursing and Health Sciences, Monash University, Victoria, Australia; Linda Kader, $\mathrm{MD}$, Orygen, The National Centre of Excellence in Youth Mental Health and the Centre for Youth Mental Health, University of Melbourne, Victoria, Australia; Saji Damodaran, MD, Michael T. H. Wong, MD, PhD, Department of Psychiatry, Faculty of Medicine, Nursing and Health Sciences, Monash University, Victoria, Australia; Philippe Conus, MD, Département Universitaire de Psychiatrie CHUV, Université de Lausanne, Clinique de Cery, Prilly, Switzerland; Aswin Ratheesh, MD, Patrick D. McGorry, MD, PhD, Sue M. Cotton, PhD, Orygen, The National Centre of Excellence
Matill in Youth Mental Health and the Centre for Youth Mental Health, University of Melbourne, Victoria, Australia

Correspondence: Michael Berk, Deakin University, IMPACT Strategic Research Centre, School of Medicine, 75 Pigdon's Road, Waurn Ponds, Geelong, Victoria, 3216, Australia. Email: mikebe@barwonhealth.org.au

First received 2 May 2016, final revision 26 Nov 2016, accepted 28 Nov 2016

\section{Funding}

This study was supported by an unrestricted grant from Astra Zeneca. M.Y. is supported by a National Health and Medical Research Council (NHMRC) Senior Research Fellowship 1021973 and Principal Research Fellowship (1117188). S.M.C. is supported by a NHMRC Career Development Fellowship 1061998. M.B. is supported by a NHMRC Senior Principal Research Fellowship 1059660. P.D.M. is supported by a NHMRC Senior Principal Research Fellowship. C.P. was supported by a NHMRC Senior Principal Research Fellowship $(628386$ and 1105825) and a Brain and Behavior Research Foundation (NARSAD) Distinguished Investigator Award (US; Grant ID: 18722) and he has received major support from NHMRC and the Australian Research Council (ARC), as well as Melbourne Health and The University of Melbourne.

\section{References}

1 Angst J, Sellaro R. Historical perspectives and natural history of bipolar disorder. Biol Psychiatry 2000; 48: 445-57.
2 Post RM, Rubinow DR, Ballenger JC. Conditioning and sensitisation in the longitudinal course of affective illness. Br J Psychiatry 1986; 149: 191-201.

3 Oepen G, Baldessarini RJ, Salvatore P, Slater E. On the periodicity of manic-depressive insanity, by Eliot Slater (1938): translated excerpts and commentary. J Affect Disord 2004; 78: 1-9.

4 Berk M, Berk L, Dodd S, Cotton S, Macneil C, Daglas R, et al. Stage managing bipolar disorder. Bipolar Disord 2014; 16: 471-7.

5 Franchini L, Zanardi R, Smeraldi E, Gasperini M. Early onset of lithium prophylaxis as a predictor of good long-term outcome. Eur Arch Psychiatry Clin Neurosci 1999; 249: 227-30.

6 Kessing LV, Vradi E, Andersen PK. Starting lithium prophylaxis early v. late in bipolar disorder. Br J Psychiatry 2014; 205: 214-20.

7 Maj M, Pirozzi R, Magliano L. Nonresponse to reinstituted lithium prophylaxis in previously responsive bipolar patients: prevalence and predictors. Am J Psychiatry 1995; 152: 1810-1.

8 Baldessarini RJ, Tondo L, Baethge CJ, Lepri B, Bratti IM. Effects of treatment latency on response to maintenance treatment in manic-depressive disorders. Bipolar Disord 2007; 9: 386-93.

9 Berk M, Dodd S. Recent developments in the treatment of bipolar disorders. Expert Opin Investig Drugs 2003; 12: 1621-32.

10 Bowden CL, Grunze $\mathrm{H}$, Mullen J, Brecher M, Paulsson B, Jones M, et al. A randomized, double-blind, placebo-controlled efficacy and safety study of quetiapine or lithium as monotherapy for mania in bipolar disorder. J Clin Psychiatry 2005; 66: 111-21.

11 McIntyre RS, Brecher M, Paulsson B, Huizar K, Mullen J. Quetiapine or haloperidol as monotherapy for bipolar mania - a 12-week, double-blind, randomised, parallel-group, placebo-controlled trial. Eur Neuropsychopharmacol 2005; 15: 573-85.

12 Vieta E, Mullen J, Brecher M, Paulsson B, Jones M. Quetiapine monotherapy for mania associated with bipolar disorder: combined analysis of two international, double-blind, randomised, placebo-controlled studies. Curr Med Res Opin 2005; 21: 923-34.

13 Calabrese JR, Keck PE Jr, Macfadden W, Minkwitz M, Ketter TA, Weisler RH, et al. A randomized, double-blind, placebo-controlled trial of quetiapine in the treatment of bipolar I or II depression. Am J Psychiatry 2005; 162 1351-60.

14 Young AH, McElroy SL, Bauer M, Philips N, Chang W, Olausson B, et al. A double-blind, placebo-controlled study of quetiapine and lithium monotherapy in adults in the acute phase of bipolar depression (EMBOLDEN I). J Clin Psychiatry 2010; 71: 150-62.

15 Keck PE, Orsulak PJ, Cutler AJ, Sanchez R, Torbeyns A, Marcus RN, et al. Aripiprazole monotherapy in the treatment of acute bipolar I mania: a randomized, double-blind, placebo- and lithium-controlled study. J Affect Disord 2009; 112: 36-49.

16 Tohen M, Ketter TA, Zarate CA, Suppes T, Frye M, Altshuler L, et al. Olanzapine versus divalproex sodium for the treatment of acute mania and maintenance of remission: a 47-week study. Am J Psychiatry 2003; 160 : 1263-71.

17 Bowden CL. Atypical antipsychotic augmentation of mood stabilizer therapy in bipolar disorder. J Clin Psychiatry 2005; 66 (suppl 3): 12-9.

18 Conus $\mathrm{P}$, Berk M, Cotton SM, Kader L, Macneil C, Hasty MK, et al. Olanzapine or chlorpromazine plus lithium in first episode psychotic mania: an 8-week randomised controlled trial. Eur Psychiatry 2015; 30 975-82.

19 First MB, Spitzer, Robert L, Gibbon Miriam, and Williams, Janet BW. Structured Clinical Interview for DSM-IV-TR Axis I Disorders, Research Version, Patient Edition. (SCID-I/P). Biometrics Research, New York State Psychiatric Institute, 2002.

20 Young RC, Biggs JT, Ziegler VE, Meyer DA. A rating scale for mania: reliability, validity and sensitivity. Br J PSychiatry 1978; 133: 429-35.

21 Overall JE, Gorham DR. The Brief Psychiatric Rating Scale. Psychol Rep 1962; 10: 799-812.

22 Berk M, Malhi GS, Cahill C, Carman AC, Hadzi-Pavlovic D, Hawkins MT, et al. The Bipolar Depression Rating Scale (BDRS): its development, validation and utility. Bipolar Disord 2007; 9: 571-9.

23 Montgomery SA, Åsberg M. A new depression scale designed to be sensitive to change. Br J Psychiatry 1979; 134: 382-9.

24 spearing M, Post RM, Leverich GS, Brandt D, Nolen W. Modification of the Clinical Global Impressions (CGI) Scale for use in bipolar illness (BP): The CGI-BP. Psychiatry Res 1997; 73: 159-71.

25 Jones SH, Thornicroft G, Coffey M, Dunn G. A brief mental health outcome scale - reliability and validity of the Global Assessment of Functioning (GAF). Br J Psychiatry 1995; 166: 654-9.

26 Morosini PL, Magliano L, Brambilla L, Ugolini S, Pioli R. Development, reliability and acceptability of a new version of the DSM-IV Social 
Occupational Functioning Assessment Scale (SOFAS) to assess routine social functioning. Acta Psychiatr Scand 2000; 101: 323-9.

27 Heinrichs DW, Hanlon TE, Carpenter WT. The Quality of Life Scale: an instrument for rating schizophrenic deficit syndrome. Schizophrenia Bull 1984; 10: 388-98.

28 Tohen M, Hennen J, Zarate Jr CM, Baldessarini RJ, Strakowski SM, Stoll AL, et al. Two-year syndromal and functional recovery in 219 cases of first episode major affective disorder with psychotic features. Am J Psychiatry 2000; 157: 220-8.

29 Mallinckrodt C, Watkin J, Molenberghs G, Carroll R. Choice of the primary analysis in longitudinal clinical trials. Pharm Stat 2004; 3: 161-9.

30 Gueorgieva R, Krystal JH. Move over ANOVA: progress in analyzing repeated-measures data and its reflection in papers published in the Archives of General Psychiatry. Arch Gen Psychiatry 2004; 61: 310-7.

31 Rothman KJ. No adjustments are needed for multiple comparisons. Epidemiology 1990; 1: 43-6.

32 Yildiz A, Vieta E, Leucht S, Baldessarini RJ. Efficacy of antimanic treatments: meta-analysis of randomized, controlled trials. Neuropsychopharmacology 2011; 36: 375-89.

33 Nierenberg AA, Sylvia LG, Leon AC, Reilly-Harrington NA, Shesler LW McElroy SL, et al. Clinical and Health Outcomes Initiative in Comparative Effectiveness for Bipolar Disorder (Bipolar CHOICE): a pragmatic trial of complex treatment for a complex disorder. Clin Trials 2014; 11: 114-27.

34 Weisler RH, Nolen WA, Neijber A, Hellqvist A, Paulsson B, Trial 144 Study Investigators. Continuation of quetiapine versus switching to placebo or lithium for maintenance treatment of bipolar I disorder (Trial 144: a randomized controlled study). J Clin Psychiatry 2011; 72: 1452-64.
35 Suppes T, Vieta E, Gustafsson U, Ekholm B. Maintenance treatment with quetiapine when combined with either lithium or divalproex in bipolar I disorder: analysis of two large randomized, placebo-controlled trials. Depress Anxiety 2013; 30: 1089-98.

36 Hartong EG, Moleman P, Hoogduin CA, Broekman TG, Nolen WA, LitCar G. Prophylactic efficacy of lithium versus carbamazepine in treatment-naive bipolar patients. J Clin Psychiatry 2003; 64: 144-51.

37 Berk M, Kapczinski F, Andreazza AC, Dean OM, Giorlando F, Maes M, et al. Pathways underlying neuroprogression in bipolar disorder: focus on inflammation, oxidative stress and neurotrophic factors. Neurosci Biobehav Rev 2011; 35: 804-17.

38 Passos IC, Mwangi B, Vieta E, Berk M, Kapczinski F. Areas of controversy in neuroprogression in bipolar disorder. Acta Psychiatr Scand 2016; 134: 91-103.

39 Calabrese JR, Vieta E, El-Mallakh R, Findling RL, Youngstrom EA, Elhaj O, et al. Mood state at study entry as predictor of the polarity of relapse in bipolar disorder. Biol Psychiatry 2004; 56: 957-63.

40 Vieta E, Berk M, Wang W, Colom F, Tohen M, Baldessarini RJ. Predominant previous polarity as an outcome predictor in a controlled treatment trial for depression in bipolar I disorder patients. J Affect Disord 2009; 119: 22-7.

41 Schalkwijk S, Undurraga J, Tondo L, Baldessarini RJ. Declining efficacy in controlled trials of antidepressants: effects of placebo dropout. Int J Neuropsychopharmacol 2014; 17: 1343-52.

42 Grande I, Berk M, Birmaher B, Vieta E. Bipolar disorder. Lancet 2016; 387 1561-72.

\section{poems \\ by doctors}

\section{Me of You}

\section{Qasim ljaz}

So here I am in the middle way

still grappling with the void,

as fresh as spring flowers,

as jaded as autumn leaves

of what belonged to you

of us -

now misted in time.

Your presence -

confident and affirmative

that sparked a thousand flames and turns

of joy and wonder

now inhabits

the sightless recesses

of my mind

and distant space -

a chimera

of half formed images and sounds,

lost in abstracted pain and longing.

Tell me where

do I begin to grieve. .

for what is left of me

that belonged to you,

stolen by a pitiless fate.

(c) Qasim ljaz 2017. Reproduced with permission. 